$4-14-2020$

\title{
Quasi-Likelihood Ratio Tests for Homoscedasticity in Linear Regression
}

\author{
Lili Yu \\ Georgia Southern University, lyu@georgiasouthern.edu \\ Varadan Sevilimedu \\ Georgia Southern University \\ Robert Vogel \\ Georgia Southern University \\ Hani Samawi \\ Georgia Southern University
}

Follow this and additional works at: https://digitalcommons.wayne.edu/jmasm

Part of the Applied Statistics Commons, Social and Behavioral Sciences Commons, and the Statistical Theory Commons

\section{Recommended Citation}

Yu, L., Sevilimedu, V., Vogel, R., \& Samawi, H. (2019). Quasi-likelihood ratio tests for homoscedasticity in linear regression. Journal of Modern Applied Statistical Methods, 18(1), eP2845. doi: 10.22237/jmasm/ 1556669460

This Regular Article is brought to you for free and open access by the Open Access Journals at DigitalCommons@WayneState. It has been accepted for inclusion in Journal of Modern Applied Statistical Methods by an authorized editor of DigitalCommons@WayneState. 


\section{Quasi-Likelihood Ratio Tests for Homoscedasticity in Linear Regression}

\author{
Lili Yu \\ Georgia Southern University \\ Statesboro, GA \\ Robert Vogel \\ Georgia Southern University \\ Statesboro, GA
}

\author{
Varadan Sevilimedu \\ Georgia Southern University \\ Statesboro, GA \\ Hani Samawi \\ Georgia Southern University \\ Statesboro, GA
}

Two quasi-likelihood ratio tests are proposed for the homoscedasticity assumption in the linear regression models. They require few assumptions than the existing tests. The properties of the tests are investigated through simulation studies. An example is provided to illustrate the usefulness of the new proposed tests.

Keywords: Bootstrap, homoscedasticity test, quasi-likelihood, regression model, variance function

\section{Introduction}

Homoscedasticity or constant variance is a standard assumption in regression models. The violation of this assumption can lead to inefficient estimation or incorrect inference (Ruppert et al., 2003). Former research studied homoscedasticity tests in different parametric and nonparametric regression models. For the linear regression model, Cook and Weisberg (1983) proposed a score test statistic for parametric variance functions; Breusch and Pagan (1979) proposed a Lagrange multiplier test for a fairly wide class of alternative hypotheses; Diblasi and Bowman (1997) constructed a nonparametric test of constant variance. All of them require the assumption that the errors have the normal distribution. Koenker (1981) proposed a variant of the Breusch-Pagan test by relaxing the normality assumption, but the alternative hypotheses still cannot include all of the possible heteroscedastic models.

doi: 10.22237/jmasm/1556669460 | Accepted: July 6, 2018; Published: April 14, 2020.

Correspondence: Lili Yu, lyu@georgiasouthern.edu 


\section{YU ET AL}

For nonparametric regression models, Eubank and Thomas (1993), Müller and Zhao (1995), respectively, presented tests for nonparametric and semiparametric variance functions under the assumption of normal distributed errors; Dette and Munk (1998), Dette (2002), Liero (2003), and Wang and Zhou (2005) developed tests by comparing variance estimators under two hypotheses under the Lipschitz continuous assumption; Francisco-Fernández and Vilar-Fernández (2008) proposed two new tests based on nonparametric smoothing; Dette et al. (2007) and Dette and Hetzler (2008) considered tests of a parametric form of conditional variance. For partially linear regression models, You and Chen (2005), Lin and Qu (2012), respectively, presented heteroscedasticity tests based on the research of Dette and Munk (1998) and Dette (2002); Dette and Marchlewski (2008) considered testing a parametric form of the conditional variance.

Linear regression models are the most popular models. The homoscedasticity tests for linear models in the literature referenced above require assumptions, such as normality and parametric variance functions. These assumptions greatly restrict their applications to real data analysis.

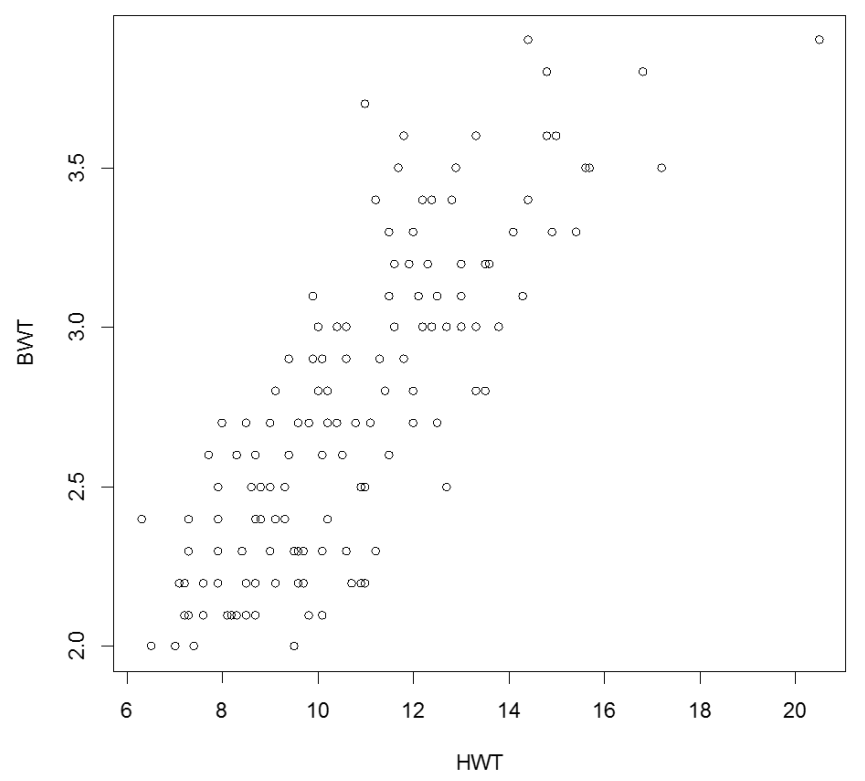

Figure 1. Scatter plot of BWT versus HWT 


\section{TESTS FOR HOMOSCEDASTICITY}

As an example, consider the Cats data in MASS package in R. 144 adult [over $2 \mathrm{~kg}$ (kilograms) in weight] cats used for experiments with the drug digitalis had their heart in $\mathrm{g}$ (grams) and body weight in $\mathrm{kg}$ recorded. The interest is if the heart weight (HWT) is associated with the body weight (BWT) for cats. A scatter plot of BWT versus HWT is given in Figure 1, which indicates a linear relationship. A linear regression line can be fit to investigate their association. However, the variances of the observations may be different. In order to choose appropriate inference methods to obtain efficient estimation or correct inference, check the homoscedasticity assumption for the linear regression model. Shown in Figure 1. the variances are larger for the middle response values than small and large response values. This suggests the researchers to consider the variance as a function of the mean. However, it is hard to specify the form of the variance function, which is required by the existing homoscedasticity tests for the linear regression model. As will be shown, if the true variance function is not in the specified alternative, the test is not reliable, i.e., cannot maintain the probability of the type-I error and has low power. Therefore, in order to obtain the reliable test results, homoscedasticity tests are proposed in which the alternative hypothesis includes all possible heteroscedastic models, for the linear regression model.

Two powerful quasi-likelihood ratio tests are proposed for linear regression models with minimum assumptions, that is, they do not require a known distribution and specific variance function forms of the data. This is achieved due to the good properties of quasi-likelihood. As shown in Wedderburn (1974), the quasi-likelihood has similar properties as the log-likelihood function. It only requires assumptions on the first two moments which is much easier to postulate than the entire distribution of the data. Due to these properties, quasi-likelihood ratio tests have the potential to achieve high power but require fewer assumptions. In addition, these tests can be easily extended to more complex models, such as partially linear models and nonparametric models, with minimum assumptions.

\section{Two Quasi-Likelihood Ratio Tests for Homoscedasticity}

Consider the linear model

$$
y_{i}=\mu_{i}+\varepsilon_{i}, \quad i=1, \ldots, n
$$

where $\varepsilon_{i}$, are identically and independently distributed with mean zero and unknown variances $\sigma^{2}\left(\mu_{i}\right) ; y_{i}$ has mean $\mu_{i}=\mathbf{X}_{i}^{\mathrm{T}} \boldsymbol{\beta}$ where $\mathbf{X}_{i}$ is a $p$-dimensional vector of known 


\section{YU ET AL}

constants and $\boldsymbol{\beta}$ is a vector of parameters. The homoscedasticity test for model (1) is to test if the variance $\sigma^{2}\left(\mu_{i}\right)$ is constant.

$$
\begin{aligned}
& \mathrm{H}_{0}: \sigma^{2}\left(\mu_{i}\right)=\sigma^{2} \text { for } i=1, \ldots, n \\
& \mathrm{H}_{1}: \sigma^{2}\left(\mu_{i}\right) \neq \sigma^{2} \text { for at least one } i=1, \ldots, n
\end{aligned}
$$

for some unknown positive constant $\sigma^{2}$.

\section{First Test: Quasi-Likelihood Ratio Test}

Quasi-likelihood proposed by Wedderburn (1974) is defined as follows:

$$
Q=\frac{1}{n} \sum_{i=1}^{n} \int_{y_{i}}^{\mu_{i}} \frac{y_{i}-a}{\sigma^{2}(a)} d a .
$$

In (2), it is assumed the variance is a function of the mean, but the form of the variance function is not specified. Therefore, few assumptions are required for the proposed tests based on the quasi-likelihood. The score function of the quasilikelihood (2) is derived as its first derivative as

$$
\mathbf{U}_{\boldsymbol{\beta}}=\frac{1}{n} \sum_{i=1}^{n} \mathbf{X}_{i}^{\mathrm{T}}\left(y_{i}-\mathbf{X}_{i}^{T} \boldsymbol{\beta}\right) / \sigma^{2}\left(\mu_{i}\right)
$$

Wedderburn (1974) showed that quasi-likelihood (2) has similar properties to the log-likelihood. Then the test statistic is constructed as

$$
Q L R_{1}=-2\left(Q_{0}-Q_{1}\right)
$$

where $Q_{0}$ is the quasi-likelihood function under $\mathrm{H}_{0}$ and $Q_{1}$ is the quasi-likelihood function under $\mathrm{H}_{1}$. Next, we will explain how to calculate these quasi-likelihood functions.

To calculate $Q_{0}$ under $\mathrm{H}_{0}$ (i.e., the data are homoscedasticity), we estimate $\boldsymbol{\beta}$ by the least squares estimator $\tilde{\boldsymbol{\beta}}$ for model (1). Then we calculate $\tilde{\mu}_{i}=\mathbf{X}_{i}^{\mathrm{T}} \tilde{\boldsymbol{\beta}}$ and $\tilde{\sigma}^{2}=1 / n \sum_{i=1}^{n}\left(y_{i}-\tilde{\mu}_{i}\right)^{2}$. Therefore, the quasi-likelihood under $\mathrm{H}_{0}$ can be calculated as 


\section{TESTS FOR HOMOSCEDASTICITY}

$$
Q_{0}=\frac{1}{n} \sum_{i=1}^{n} \int_{y_{i}}^{\tilde{\mu}_{i}} \frac{y_{i}-a}{\tilde{\sigma}^{2}} d a .
$$

To calculate $Q_{1}$ under $\mathrm{H}_{1}$ (i.e., the data are heteroscedasticity), we first estimate $\boldsymbol{\beta}$ by maximizing the quasi-likelihood (2), which is same as solving the score function $\mathbf{U}_{\boldsymbol{\beta}}=0$. In $\mathbf{U}_{\boldsymbol{\beta}}$, we approximate the variance function $\sigma^{2}\left(\mu_{i}\right)$ as a spline function. Specifically, the variance function is approximated by a basis expansion

$$
\sigma^{2}\left(\mu_{i}\right)=\sum_{j=1}^{J} \mathrm{~B}_{j}\left(\mu_{i}\right) \gamma_{j},
$$

where $J$ is the number of $B$-spline basis functions, $\mathrm{B}_{j}, j=1, \ldots, J$, are the $B$-spline basis functions (De Boor, 2001) and $\gamma_{j}, j=1, \ldots, J$, are unknown spline coefficients to be estimated. The number $J$ is determined by the degree of the splines and the number of knots, the choices of which will be introduced in the following algorithm. Then the score function is approximated by

$$
\tilde{\mathbf{U}}_{\boldsymbol{\beta}}=\frac{1}{n} \frac{\sum_{i=1}^{n} \mathbf{X}_{i}^{T}\left(y_{i}-\mu_{i}\right)}{\sum_{j=1}^{J} \mathrm{~B}_{j}\left(\mu_{i}\right) \gamma_{j}},
$$

which is a function of $\mu_{i}$. Therefore, the estimator $\hat{\boldsymbol{\beta}}$ is the solution of $\tilde{\mathbf{U}}_{\boldsymbol{\beta}}=0$. We use the following algorithm to obtain $\hat{\boldsymbol{\beta}}$ and calculate the quasi-likelihood under $\mathrm{H}_{1}$ :

(1) Initialize the variances with $\hat{\sigma}^{2(0)}\left(\mu_{i}\right)=1$.

(2) At the $(k+1)^{\text {th }}$ step,

(a) For the given variance $\hat{\sigma}^{2(k)}\left(\mu_{i}\right)$, the parameter estimates $\hat{\boldsymbol{\beta}}^{(k+1)}$ are obtained by maximizing the quasi-likelihood (2) using the Newton-Raphson with scoring method.

(b) For given $\hat{\boldsymbol{\beta}}^{(k+1)}$, calculate $\hat{\mu}_{i}=\mathbf{X}_{i}^{\mathrm{T}} \hat{\boldsymbol{\beta}}^{(k+1)}$ and the basis expansions $\mathrm{B}_{j}\left(\hat{\mu}_{i}\right)$. Then the estimators $\hat{\gamma}_{j}$ of $\gamma_{j}$ are obtained by the least squares method for the following model: 


\section{YU ET AL}

$$
\hat{\varepsilon}_{i}^{2}=\sum_{j=1}^{J} \mathrm{~B}_{j}\left(\hat{\mu}_{i}\right) \gamma_{j}+\phi_{i}
$$

where $\hat{\varepsilon}_{i}^{2}=\left(y_{i}-\hat{\mu}_{i}\right)^{2}$ are the 'observed' values of the variances $\sigma^{2(k+1)}\left(\mu_{i}\right)$ and $\phi_{i}, i=1, \ldots, n$, are error terms with mean zero and constant variance. Now $\sigma^{2(k+1)}(a)$ is calculated by $\hat{\sigma}^{2(k+1)}(a)=\sum_{j=1}^{J} \mathrm{~B}_{j}(a) \hat{\gamma}_{j}$, where $J=n_{\text {knots }}+d-1$ and $n_{\text {knots }}$ and $d$ are the number of knots and the degree of the spline, respectively. In the algorithm, we use a cubic $B$-spline, so $d=3$, and the bs function in R software is used to choose the quantiles as knots for a given number of knots. The number of knots is varied in the range from 1 to 15 , out of which we select the one that minimizes the Akaike-like (AIC-like) criterion (Yu \& Peace, 2012)

$$
\text { AIC-like }=\sum_{i=1}^{n} \frac{y_{i}-\hat{\mu}_{i}}{\hat{\sigma}^{2(k+1)}\left(\hat{\mu}_{i}\right)}+2\left(n_{\text {knots }}+d-1\right) .
$$

(c) Repeat Steps (a)-(b) until $\left|\hat{\boldsymbol{\beta}}^{(k+1)}-\hat{\boldsymbol{\beta}}^{(k)}\right|<l\left|\hat{\boldsymbol{\beta}}^{(k+1)}\right|$, where $l$ is the prespecified convergence criterion. The converged estimators are $\hat{\boldsymbol{\beta}}$ and the corresponding variance estimators are $\hat{\sigma}^{2}($.$) . Chiou$ and Müller (1999) proved that under regularity conditions, both $\hat{\boldsymbol{\beta}}$ and $\hat{\sigma}^{2}($.$) are consistent estimators.$

(3) The quasi-likelihood under $\mathrm{H}_{1}$ is calculated as

$$
Q_{1}=\frac{1}{n} \sum_{i=1}^{n} \int_{y_{i}}^{\hat{\mu}_{i}} \frac{y_{i}-a}{\hat{\sigma}^{2}(a)} d a
$$

Now we are ready to calculate the quasi-likelihood ratio test statistic as

$$
Q L R_{1}=-2 \times \frac{1}{n}\left(\sum_{i=1}^{n} \int_{y_{i}}^{\tilde{\mu}_{i}} \frac{y_{i}-a}{\tilde{\sigma}^{2}} d a-\sum_{i=1}^{n} \int_{y_{i}}^{\hat{\mu}_{i}} \frac{y_{i}-a}{\tilde{\sigma}^{2}(a)} d a\right) .
$$




\section{Second Test: Modified Quasi-Likelihood Ratio Test}

A modified quasi-likelihood is proposed:

$$
Q^{*}=\frac{1}{n} \sum_{i=1}^{n} \int_{y_{i}}^{\mu_{i}} \frac{y_{i}-a}{\sigma^{2}\left(\mu_{i}\right)} d a .
$$

It is easy to see that (3) has the same first derivative as (2). In other words, (3) and (2) have the same score functions. Then (3) has similar properties to the log likelihoods as well based on the Theorem 1 in Wedderburn (1974, p. 440). Therefore, it can be used to construct likelihood ratio test as well. Moreover, the estimators of $\boldsymbol{\beta}$ are same by maximizing (3) or (2) because they have the same score functions.

The motivation of the modified quasi-likelihood is that it has the same value under $\mathrm{H}_{0}$ as (2). In addition, the calculation for (3) is much less time consuming than the calculation for (2). First, we only need to calculate the variances at $\hat{\mu}_{i}$ values for (3) but need to calculate all values in the integral for (2). Second, the integration of (3) has a closed form. However, (2) does not have a closed form and we need approximations in the calculation.

The test statistic based on (3) is constructed as

$$
Q L R^{*}=-2\left(Q_{0}^{*}-Q_{1}^{*}\right)
$$

where $Q_{0}^{*}$ is the modified quasi-likelihood function under $\mathrm{H}_{0}$ and $Q_{1}^{*}$ is the modified quasi-likelihood function under $\mathrm{H}_{1}$. Because the estimators of $\boldsymbol{\beta}$ based on (3) are same as those based on (2) under both $\mathrm{H}_{0}$ and $\mathrm{H}_{1}$, use the same procedures to estimate the $\boldsymbol{\beta}$ and $\sigma^{2}\left(\mu_{i}\right)$. Then

$$
Q_{0}^{*}=Q_{0}=\frac{1}{n} \sum_{i=1}^{n} \int_{y_{i}}^{\tilde{\mu}_{i}} \frac{y_{i}-a}{\tilde{\sigma}^{2}} d a
$$

and

$$
Q_{1}^{*}=\frac{1}{n} \sum_{i=1}^{n} \int_{y_{i}}^{\tilde{\mu}_{i}} \frac{y_{i}-a}{\hat{\sigma}^{2}\left(\hat{\mu}_{i}\right)} d a .
$$




\section{YU ET AL}

The quasi-likelihood ratio test statistic is

$$
\begin{aligned}
Q L R_{2} & =-2 * \frac{1}{n}\left(\sum_{i=1}^{n} \int_{y_{i}}^{\tilde{\mu}_{i}} \frac{y_{i}-a}{\tilde{\sigma}^{2}} d a-\sum_{i=1}^{n} \int_{y_{i}}^{\hat{\mu}_{i}} \frac{y_{i}-a}{\hat{\sigma}^{2}\left(\hat{\mu}_{i}\right)} d a\right) \\
& =\frac{1}{n}\left(\frac{1}{\tilde{\sigma}^{2}} \sum_{i=1}^{n}\left(y_{i}-\tilde{\mu}_{i}\right)^{2}-\sum_{i=1}^{n} \frac{1}{\hat{\sigma}^{2}\left(\hat{\mu}_{i}\right)}\left(y_{i}-\hat{\mu}_{i}\right)^{2}\right)
\end{aligned}
$$

Remark. Comparing $Q L R_{1}$ and $Q L R_{2}$, we see that the only difference between them is the denominator $\hat{\sigma}^{2}($.$) of quasi-likelihood under \mathrm{H}_{1}$. For different patterns of the variance functions, either one of the proposed tests can be more powerful than the other one.

\section{Bootstrap Procedure for the Critical Values of the Test}

Because the quasi-likelihood has the properties of log likelihood, the proposed quasi-likelihood ratio tests follow the Chi-square distribution based on the theory of the likelihood ratio tests. However, it is well known that for nonparametric smoothing tests, the asymptotic theory does not provide accurate approximations to the distribution of the test statistic when the sample size is small or moderate (Hardle \& Mammen, 1993). Alternatively, bootstrap method is often used to approximate the distribution of the test statistic. Therefore, a similar bootstrap procedure as in Wang and Zhou (2005) is proposed to approximate the critical values of the tests in practical applications. The bootstrap procedure is proposed as follows. Denote $B$ as the sufficient large number of bootstrapping. Therefore, for $b=1, \ldots, B$,

(1) For $i=1, \ldots, n$, let $y_{i, b}^{*}=\mathbf{X}_{i}^{\mathrm{T}} \tilde{\boldsymbol{\beta}}+\varepsilon_{i}^{*}$, where $\varepsilon_{i}^{*}$ is a bootstrap sample from centered $\tilde{\varepsilon}_{i}=\left(y_{i}-\tilde{\mu}_{i}\right)$.

(2) With the bootstrap sample $\left\{\left(\mathbf{X}_{i}, y_{i, b}^{*}\right), i=1, \ldots, n\right\}$, calculate $Q L R_{1 b}$ and $Q L R_{2 b}$ for each bootstrapping sample using the new proposed methods.

(3) Let $Q L R_{1 \eta}$ be the $\eta^{\text {th }}$ order statistic of $Q L R_{11}, \ldots, Q L R_{1 B}$; then $Q L R_{1|(1-\alpha) B|}$ approximates the $(1-\alpha)$-quantile of the distribution of $Q L R_{1}$ under the null hypothesis. Similarly, calculate $Q L R_{2|(1-\alpha) B|}$ to approximate the $(1-\alpha)$-quantile of the distribution of $Q L R_{2}$ under the null hypothesis. 


\section{TESTS FOR HOMOSCEDASTICITY}

\section{Simulations}

The performance of the proposed tests is investigated for finite samples. Compare the two quasi-likelihood ratio tests with the parametric score test proposed by Cook and Weisberg (CW test) (Cook \& Weisberg, 1983), the Breusch-Pagan test (BP test) (Breusch \& Pagan, 1979), and its variant proposed by Koenker (VBP test) (Koenker, 1981). These tests require assumptions, such as normal error and some specific functional forms of the variance.

\section{Evaluate Size (Type-I Error) of the Test}

Homoscedastic data is generated under $\mathrm{H}_{0}$ from $y_{i}=1+x_{i}+\varepsilon_{i}$, where the $x_{i}$ are generated from the standard normal distribution. Consider two different distributions for $\varepsilon_{i}$ : the standard normal distribution $[\mathrm{N}(0,1)]$, and the $t$ distribution with 4 degree of freedom [t(4)]. Sample sizes are 70 and 100. The test is calculated with 1000 simulation runs for each scenario and nominal level 0.05 . We use $B=500$ bootstrap samples per run to obtain the critical values. The results are summarized in Table 1 and it can be seen that the proposed tests maintain the specified nominal level satisfactory. For $\mathrm{CW}$ and $\mathrm{BP}$ tests, when the error term follows $t$ distribution, the probability of type-I errors is much higher or lower than the nominal level 0.05. Clearly, CW and BP tests are not robust. However, VBP test is robust for the non-normal distributed data.

\section{Empirical Power of the Test}

In order to investigate the power of the tests, consider two alternatives:

Alternative I: $\quad y_{i}=1+x_{i}+0.5 \exp \left(2 x_{i}\right) \varepsilon_{i}$

Alternative II: $\quad y_{i}=1+x_{i}+0.5\left[1+\sin \left(3 x_{i}\right)\right] \varepsilon_{i}$

where both $x_{i}$ and $\varepsilon_{i}$ are generated from the standard normal distribution. Table 2 summarizes the results. Alternative I satisfies all of the assumptions required by the $\mathrm{CW}, \mathrm{BP}$, and VBP tests, and they are more powerful than the quasi-likelihood ratio tests. However, in alternative II, the true form of the variance function does not fit the general formulation of the alternative hypotheses in the CW, BP, and VBP tests, and their powers are much lower than the new proposed quasi-likelihood ratio tests. The power of the quasi-likelihood ratio tests is satisfactory. The first quasilikelihood ratio test is more powerful than the second test for alternative I, but less powerful for alternative II. Although the classical tests are powerful against the 


\section{YU ET AL}

Table 1. Empirical size of the tests

\begin{tabular}{rrrr}
$\boldsymbol{N}$ & & $\mathbf{N}(\mathbf{0}, \mathbf{1})$ & $\mathbf{t}(\mathbf{4})$ \\
\hline 70 & Test I & 0.045 & 0.040 \\
& Test II & 0.054 & 0.067 \\
& CW & 0.051 & 0.170 \\
& BP & 0.051 & 0.170 \\
& VBP & 0.044 & 0.052 \\
100 & Test I & 0.038 & 0.049 \\
& Test II & 0.063 & 0.047 \\
& CW & 0.044 & 0.193 \\
& BP & 0.044 & 0.193 \\
& VBP & 0.044 & 0.050 \\
\hline
\end{tabular}

Table 2. Empirical power of the tests

\begin{tabular}{rrrr}
$\boldsymbol{N}$ & & Alternative I & Alternative II \\
\hline 70 & Test I & 0.548 & 0.570 \\
& Test II & 0.355 & 0.964 \\
& CW & 1.000 & 0.179 \\
& BP & 1.000 & 0.179 \\
& VBP & 1.000 & 0.041 \\
100 & Test I & 0.588 & 0.848 \\
& Test II & 0.464 & 0.981 \\
& CW & 1.000 & 0.178 \\
& BP & 1.000 & 0.178 \\
& VBP & 1.000 & 0.043 \\
\hline
\end{tabular}

specified alternative, they may have very low power if the true variance forms are not in the specified direction. However, the new proposed quasi-likelihood tests have satisfactory powers for different true variance forms because they include all possible variance forms in the alternative hypothesis.

\section{Cats Data Analysis}

The usefulness of the new quasi-likelihood ratio tests is illustrated on the Cats data, which is introduced in the Introduction section. The intent is to investigate if the heart weight (HWT) is associated with the body weight (BWT) for cats. Scatter plot (Figure 1) shows the linear relationship between BWT and HWT. Therefore, the following linear regression model is fitted to the data:

$$
B W T_{i}=\beta_{0}+\beta_{1} H W T_{i}+\varepsilon_{i},
$$




\section{TESTS FOR HOMOSCEDASTICITY}

where $\varepsilon_{i}$ is the error term with mean zero and variance $\sigma^{2}\left(\mu_{i}\right)$ and $\mu_{i}=\beta_{0}+\beta_{1} H W T_{i}$. First, we check the normality assumption of the data. Q-Q plot (Figure 2) shows that the data satisfies the normality assumption. In order to see the variance form, Figure 3 shows the plot of $\hat{\varepsilon}_{i}^{2}$ versus $\hat{\mu}_{i}$, where $\hat{\varepsilon}_{i}=B W T_{i}-\hat{\beta}_{0}-\hat{\beta}_{1} H W T_{i}, \hat{\beta}_{0}, \hat{\beta}_{1}$ are the least squares estimators of $\beta_{0}, \beta_{1}$ in model (4), and $\hat{\mu}_{i}=\hat{\beta}_{0}+\hat{\beta}_{1} H W T_{i}$. It indicates the homoscedasticity assumption is not satisfied. Because the normality assumption is satisfied, the $\mathrm{CW}$ and the BP tests may be used to check the homoscedasticity assumption. Both the $\mathrm{CW}$ and the BP tests got a $p$-value 0.11 by assuming the variance of BWT $\sigma^{2}\left(\mu_{i}\right)$ is a function of the mean $\mu_{i}$. It concludes the data is not heteroscedastic.

However, as shown in Figure 3, the variance form is not exponential, which is assumed by the $\mathrm{CW}$ test, so the $\mathrm{CW}$ test has low power. For the $\mathrm{BP}$ test, although the variance form is sufficiently general to include a fairly wide class of heteroscedastic models, it does not include some heteroscedastic models, such as $\sigma_{i}^{2} \propto \mu_{i}^{2}$. From Figure 3, the square of the mean may contribute to the shape of the variance function. This may be the reason that the BP test has low power and hence is not significant. Then, apply the two quasi-likelihood tests to this linear regression model. The first test gives a $p$-value 0.03 , and the second test gives a $p$-value 0.2 . The first test is significant and the second one is not. This suggests the heteroscedastic variance structure of this dataset is easier to be identified by the

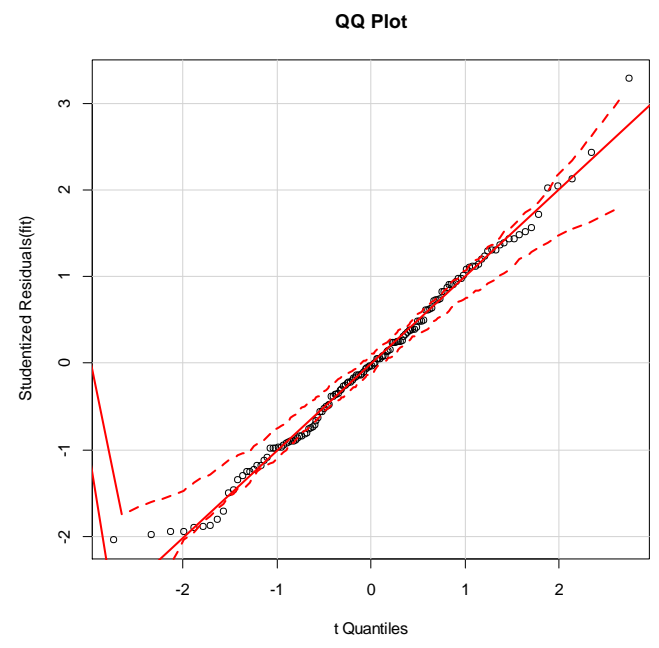

Figure 2. Q-Q plot to check the normality assumption 


\section{YU ET AL}

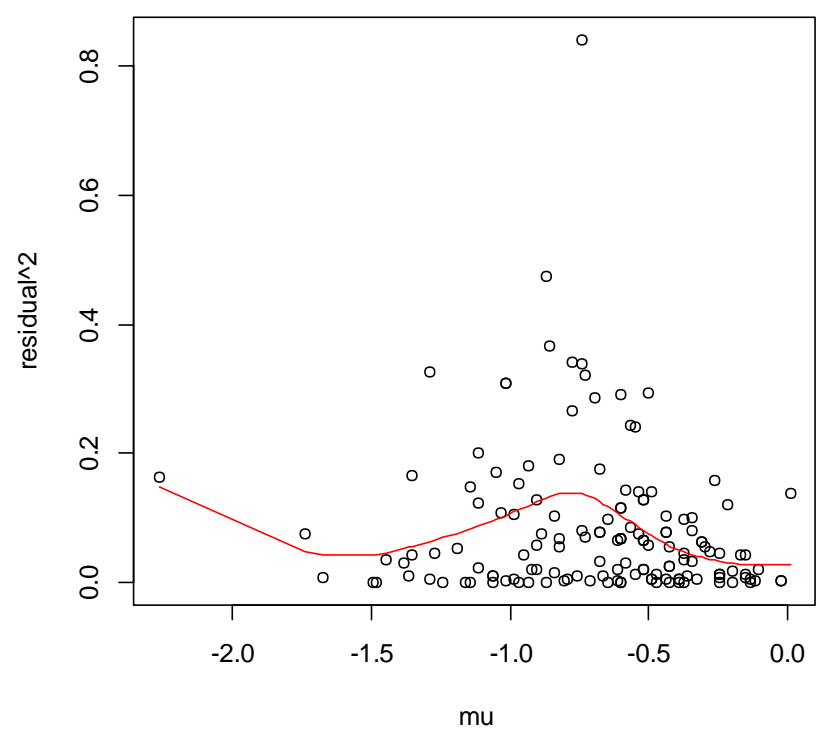

Figure 3. Plot of checking the variance function

first test than the second one, so the first test is more powerful than the second one. Because the quasi-likelihood ratio tests can test for all possible heteroscedastic models, their test results are reliable, and we conclude that the data is heteroscedastic.

\section{Discussions and Conclusions}

The classical homoscedasticity tests for linear regression models, such as the CW and the BP tests, cannot test for all possible heteroscedastic models. If the true variance form is not in the specified alternative hypotheses, the tests are not reliable. However, for real data, the true variance form is usually unknown and is hard to specify, so we do not know if the test results are valid or not. In this paper, we proposed two quasi-likelihood ratio tests, which can test for all possible heteroscedastic models. Hence, they are valid for all real data and have wider applications than the classical homoscedasticity tests for linear regression models. Moreover, the quasi-likelihood ratio tests have the potential to achieve greater power due to its log-likelihood properties. Furthermore, the quasi-likelihood ratio tests are easy to be implemented. The simulation shows the new proposed quasi- 


\section{TESTS FOR HOMOSCEDASTICITY}

likelihood ratio tests maintain the probability of type-I error well and have satisfactory powers. The real data analysis shows the new proposed tests are useful.

A new form of quasi-likelihood is proposed for the second test. It has a simpler form that results in easy and less time-consuming computations. Simulations show the quasi-likelihood ratio test based on this new form is more powerful for some scenarios. The proposed tests can be easily extended to other models by using the estimators in the quasi-likelihood for the corresponding models. Future research can be conducted to extend these two tests to other models, such as nonparametric models or partial linear models.

\section{Acknowledgements}

An earlier version of this paper was presented at the 2017 Spring Meeting of the Eastern North America Region of the International Biometric Society, Washington, DC.

\section{References}

Breusch, T. S., \& Pagan, A. R. (1979). A simple test for heteroscedasticity and random coefficient variation. Econometrica, 47(5), 1287-1294. doi:

$10.2307 / 1911963$

Chiou, J.-M., \& Müller, H.-G. (1999). Nonparametric quasi-likelihood. The Annals of Statistics, 27(1), 36-64. doi: 10.1214/aos/1018031100

Cook, D., \& Weisberg, S. (1983). Diagnostics for heteroscedasticity in regression. Biometrika, 70(1), 1-10. doi: 10.1093/biomet/70.1.1

De Boor, C. (2001). Practical guide to splines. New York: Springer.

Dette, H. (2002). A consistent test for heteroscedasticity in nonparametric regression based on the kernel method. Journal of Statistical Planning and Inference, 103(1-2), 311-329. doi: 10.1016/s0378-3758(01)00229-4

Dette, H., \& Hetzler, B. (2008). A simple test for the parametric form of the variance function in nonparametric regression. Annals of the Institute of Statistical Mathematics, 61, 861-886. doi: 10.1007/s10463-008-0169-1

Dette, H., \& Marchlewski, M. (2008). A test for the parametric form of the variance function in a partial linear regression model. Journal of Statistical Planning and Inference, 138(10), 3005-3021. doi: 10.1016/j.jspi.2007.11.007 


\section{YU ET AL}

Dette, H., \& Munk, A. (1998). Testing heteroscedasticity in nonparametric regression. Journal of the Royal Statistical Society: Series B (Methodology), 60(4), 693-708. doi: 10.1111/1467-9868.00149

Dette, H., Neumeyer, N., \& Van Keilegom, I. (2007). A new test for the parametric form of the variance function in non-parametric regression. Journal of the Royal Statistical Society: Series B (Methodology), 69(5), 903-917. doi: 10.1111/j.1467-9868.2007.00616.x

Diblasi, A., \& Bowman, A. (1997). Testing for constant variance in a linear model, Statistics \& Probability Letters, 33(1), 95-103. doi: 10.1016/s01677152(96)00115-0

Eubank, R. L., \& Thomas, W. (1993). Detecting heteroscedasticity in nonparametric regression. Journal of the Royal Statistical Society: Series B (Methodological), 55(1), 145-155. doi: 10.1111/j.2517-6161.1993.tb01474.x

Francisco-Fernández, M., \& Vilar-Fernández, J. (2008). Two tests for heteroscedasticity in non-parametric regression. Computational Statistics, 24, 145-163. doi: 10.1007/s00180-008-0110-3

Hardle, W., \& Mammen, E. (1993). Comparing nonparametric versus parametric regression fits. The Annals of Statistics, 21(4), 1926-1947. doi: 10.1214/aos/1176349403

Koenker, R. (1981). A note on Studentizing a test for heteroscedasticity. Journal of Econometrics, 17(1), 107-112. doi: 10.1016/0304-4076(81)90062-2

Liero, H. (2003). Testing homoscedasticity in nonparametric regression. Journal of Nonparametric Statistics, 15(1), 31-51. doi: 10.1080/10485250306038

Lin, J., \& Qu, X. (2012). A consistent test for heteroscedasticity in semiparametric regression with nonparametric variance function based on the kernel method. Statistics, 46(5), 565-576. doi: 10.1080/02331888.2010.543464

Müller, H.-G., \& Zhao, P.-L. (1995). On a semiparametric variance function model and a test for heteroscedasticity. The Annals of Statistics, 23(3), 946-967. doi: 10.1214/aos/1176324630

Ruppert, D., Wand, M. P., \& Carroll, R. J. (2003). Semiparametric regression. New York: Cambridge University Press. doi: $10.1017 / \mathrm{cbo} 9780511755453$

Wang, L., \& Zhou, X. (2005). A fully nonparametric diagnostic test for homogeneity of variances. Canadian Journal of Statistics, 33(4), 545-558. doi: $10.1002 /$ cjs.5550330406 


\section{TESTS FOR HOMOSCEDASTICITY}

Wedderburn, R. (1974). Quasi-likelihood functions, generalized linear models, and the Gauss-Newton method. Biometrika, 61(3), 439-447. doi: 10.1093/biomet/61.3.439

You, J., \& Chen, G. (2005). Testing heteroscedasticity in partially linear regression models. Statistics and Probability Letters, 73(1), 61-70. doi: 10.1016/j.spl.2005.03.002

Yu, L., \& Peace, K. (2012). Spline nonparametric quasi-likelihood regression within the frame of the accelerated failure time model. Computational Statistics and Data Analysis, 56(9), 2675-2687. doi: 10.1016/j.csda.2012.02.009 